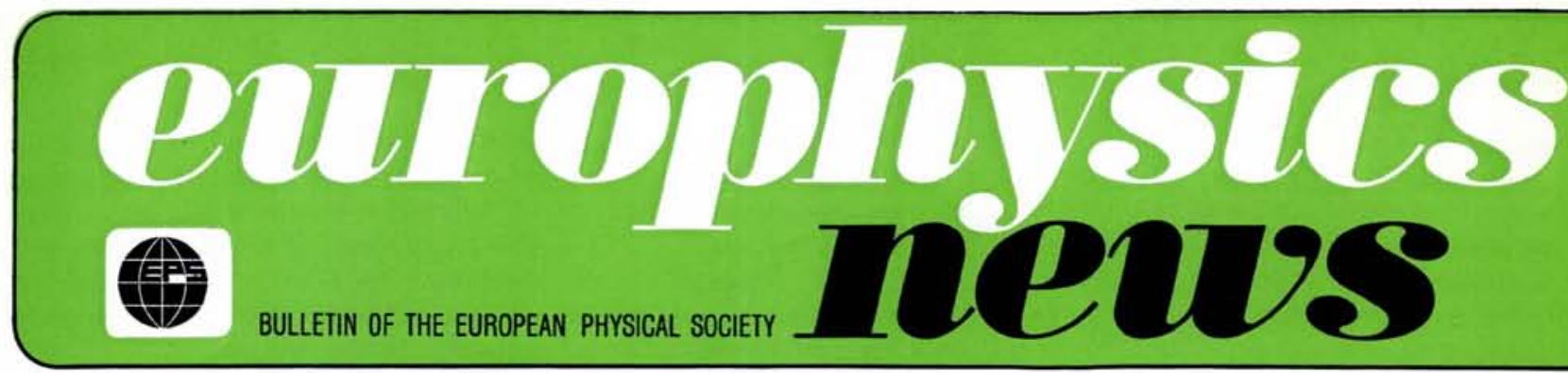

\title{
Physics in Austria
}

Physics research and teaching in Austria have grown up together, and, in the modern view of science, date back to Christian Doppler (1803-1853), Josef Stefan, the teacher of Ludwig Boltzmann, and Ernst Mach. Research in physics is performed primarily at the institutes of the Austrian Universities as well as at research institutes, especially those of the Academy of Sciences (Institute for High Energy Physics, Institute for Radium Research and Nuclear Physics, both in Vienna, ErichSchmidt Institute for Solid State Physics in Leoben, in close connection with the University of Mining there), the Institute for Solid State Physics of the Ludwig Boltzmann Society and various institutes of the Austrian Society for Studies in Atomic Energy (Seibersdorf). The "Atominstitut of the Austrian Universities" in Vienna, is a joint institution of all Austrian Universities. In the industrial sector, physics is dominated by development programmes. Here the main areas of interest are metallurgy, optical instruments and electronics.

Physics research at the Universities is financed out of the regular budget and with the help of research contracts with two foundations, one for the support of scientific research and one for applied research. Funds are granted in two ways: for individual projects, proposed and performed by small groups, and as "high priority topics" usually a joint project of several groups. During the past years one such high priority topic was high energy and elementary particle physics (now discontinued), others were plasmaphysics and semiconductor technology. High priority projects are being reconsidered at present. Generally speaking, the level of financial support for science has been raised dramatically in the past 15 years in
Austria starting from a comparatively low base line; it is now levelling off. Well proposed, small and medium scale projects have still a good chance of financial support by one of the different funds.

It is evident that a country like Austria may set up its own big experimental installations in a limited way only. The Atominstitut of the Austrian Universities has a $250 \mathrm{~kW}$ Triga reactor at its disposal and the Austrian Society for Studies in Atomic Energy runs a swimming-pool reactor with a power output of $8 \mathrm{MW}$. At the Technical University in Graz there is a small Argonaut reactor. It is therefore clear that Austrian physicists seek to undertake experiments at more powerful and bigger installations through international cooperations.

Austria is a member of CERN and a valuable cooperation has been established by the Academy of Sciences with SIN, extending significantly the experimental possibilities in the field of subatomic physics. Austria's efforts in this area are not without tradition. The present Institute for Radium Research and Nuclear Physics was founded already in 1910 and has been making recognized contributions ever since. Among its most distinguished members during the pioneering days of nuclear and elementary particle physics we should like to mention the names of such scientists as St. Meyer, V. F. Hess, M. Blau. At present, access to intense neutron sources is being sought through international cooperation, in order to extend the experience obtained already in Austria on a smaller scale.

Apart from very official projects of international cooperation almost every institute has friendly and professional contacts with numerous colleagues in the neighbouring countries. Fruitful
H. Heinrich, Linz N. Pucker, Graz (University)

scientific work is done without bureaucratic regulations and with relatively small expenditure. An outstanding example in this area is the so-called "Triangle Collaboration" in elementary particle physics, which had its origin in an almost completely informal, but nevertheless intensive cooperation between the Universities of Vienna, Bratislava and Budapest. Today the Triangle Collaboration encompasses, besides elementary particle physicists of the above mentioned Universities, also physicists in Graz, Prague, Trieste and Zagreb. The scientific value of this collaboration is highly rated and it has been recommended by UNESCO (and others) as a model for regional scientific cooperation.

One of the firmly established events in the scientific calendar is the Schladming-Winterschool in Styria. It was founded in 1962 under the patronage of the University of Graz and subsequently acqired an international reputation through the efforts of $P$. Urban, who is also the editor of Acta Physica Austriaca.

\section{Contents}

Physics in Austria

Research at Brown Boveri

Nobel Prize for 1978

P. L. Kapitza

Council Report from Rome

EPS Scholarships

EPS Lecturer Exchange

Scheme

Dedication of PETRA

Solar Physics Workshop

European Great Projects:

Errata and Addenda .

1979 Hewlett-Packard

Europhysics Prize

New Individual Ordinary

and Associate Members 
A statistical survey, conducted several years ago, shows that solid state physics is represented very strongly in Austria. It is followed by elementary particle and high energy physics, nuclear physics, atomic and molecular physics. In the following, a few illustrative examples of research areas are described, starting with the high priority topic plasmaphysics.

The research activities connected with plasma physics had their origin in the studies of the plasma theory group at the University of Innsbruck, which is still active in investigations of theoretical aspects of the project, especially plasma instabilities and plasma confinement by magnetic fields. From this beginning a substantial expansion took place directed towards experimental investigations at both the University of Innsbruck and the University of Technology in Vienna. In the coming years, besides macroscopic plasma phenomena, diagnostic methods and elementary processes will be especially studied. Topics of interest are the investigation of instabilities and the propagation of plasma waves as well as the study of ion-molecule reactions, the ionization cross-sections and the interaction of energetic ions with surfaces. Through cooperations with a number of institutions abroad these investigations are tied into the international network of plasma research.

Solid state physics is represented in Austria primarily by the fields of metal physics and semi-conductor physics as well as by the development oriented activities in metallurgy. These last are concentrated in the laboratories of Voest/Alpine (associated Austrian steel industry) and partly at the Erich Schmidt Institute in Leoben as well as in Seibersdorf. Some of the main interests in metal physics are studies of mechanical properties on a macroscopic and microscopic scale, electron microscopy, magnetic properties and band structure. This work is under way in Vienna (University and University of Technology), Seibersdorf, Graz and Leoben. Recent results include investigations and theoretical analysis of dynamics of Lomer-Cottrell dislocations eg. in $\mathrm{Al}(\mathrm{Cu})$. Scanning electron microscopy is used in connection with colour displays for distribution of elements as well as cathodoluminescence.

Semiconductor physics as a major effort is of relatively recent origin in Austria and started with the foundation of the Ludwig Boltzmann Institute for Solid State Research in Vienna fifteen years ago. At present, active groups in this field exist at the Universities in
Vienna, Graz and Linz. Research is being performed on the influence of hot electrons on MOS-structures and also in the field of non-linear conductivity in quasi one-dimensional conductors ( $\mathrm{NbSe}_{3}, \mathrm{TTF}-\mathrm{TCNQ}, \mathrm{KCP}, \mathrm{CH}_{\times}$). Tunable far infrared emission from inversion layers in silicon has been obtained recently. A research programme on narrow gap IV-VI compounds includes hot-wall epitaxial growth of crystals, defect level spectroscopy and lattice dynamics. Further fields of interest are transport effects, optical properties especially in the far infrared region and Raman spectroscopy, as well as electron-phonon interaction. Practically all groups cooperate in an application oriented high priority topic project. The goals set are far infrared sources from Landau emission, near infrared devices and application of semiconductors in medicine and application in telecommunications.

The Institute of High Energy Physics in Vienna is the centre of experimental high energy physics research in Austria. Primarily such research is performed in collaboration with the experimental programme of CERN, but also some investigations have been carried out at Serpukhov (USSR). The cooperation follows the established scheme that the development of the necessary soft- and hardware for the experiments and the data evaluation is done in Vienna (eg. multi-wire proportional chambers and highly specialized electronics). Special interest is focused on scattering experiments with hadrons (K-mesons and protons). Currently, physicists of the Institute for High Energy Physics are also collaborating with the proton-antiproton experiment designed to detect the intermediate vector bosons $\mathrm{W} \pm$ and $Z^{0}$. Theoretical studies in high energy and elementary particle physics are carried out also at the University of Vienna, Graz and Innsbruck and cover a wide range of problems.

Through the Institute of Radium Research and Nuclear Physics, the Academy of Sciences contributes to an important part of experimental nuclear physics. A further substantial institution in this area is the Atominstitut of the Austrian Universities. Groups are looking forward to the continuation of their cooperation with SIN for investigations in the medium energy range especially for neutron-neutron interaction, fission of highly excited nuclei ${ }^{238} \mathrm{U},{ }^{232} \mathrm{Th}$ ) by $\alpha$-particles and threenucleon transfer reactions. Precision measurements of neutron masses and study of neutron reactions in cooperation with the Munich Tandem Acce- lerator Institute are presently performed. In applied nuclear physics, research is performed in activation analysis, nuclear medicine and dating by ${ }^{14} \mathrm{C}$ and other methods. An extensive programme of research in nuclear data with $14 \mathrm{MeV}$ neutrons is being carried out especially with respect to neutron reactions in future fusion reactors, thereby cooperating with the international nuclear data evaluation. In addition investigations of nuclear charge distributions (Atominstitut of the Austrian Universities) and very detailed studies of the nucleon-nucleon interaction and the three-body problem (University of Graz) have to be mentioned. Applications of nuclear physics, belonging to problems of nuclear technology, are dealt with by the Austrian Society for Studies in Atomic Energy (Seibersdorf).

In order to produce an adequate impression of the varied physics research areas in Austria, the few selected areas which have been described in some detail above should, however, be supplemented by mentioning the research projects of further groups, which work on a wide range of problems, comprising mathematical physics, laser research, thin films and surfaces, atomic physics as well as the application of physical methods to medical therapy and diagnostics.

The Austrian Physical Society - a member of EPS - represents about 600 members. At present the following subsections are established: Optics, Nuclear Physics, Solid State Physics, Industrial Physics, Education. An annual meeting is held in the autumn, often in cooperation with the physical societies of one of the neighbouring countries. This year's meeting will be held in UIm together with the DPG and the SPG.

Austria always had - and still has - impressive resources of well trained young physicists: unfortunately many of them have left the country in the past. In one way or another Schrödinger and Pauli can be considered as the most prominent representatives of this large group. It is an important question, still to be solved, how to incorporate the young people in the process of research and development especially in the industrial sector. The solution of the problem will not be a simple matter of money and funding - although these play a part particularly in the typical Austrian situation of primarily small and medium sized companies. What is needed first are good ideas, more courage and daring on each side and certainly a good coordination by an appropriate agency. 